\title{
Astrophysical significance of the detection of coalescing binaries with gravitational waves
}

\author{
T. Bulik ${ }^{1}$, K. Belczyński ${ }^{2,3}$, and B. Rudak ${ }^{1}$ \\ 1 Nicolaus Copernicus Astronomical Center, Bartycka 18, 00716 Warsaw, Poland \\ 2 Northwestern University, 2145 Sheridan Rd. Evanston, IL, USA \\ 3 Lindheimer Postdoctoral Fellow
}

Received 11 July 2003 / Accepted 12 September 2003

\begin{abstract}
We use the StarTrack stellar population synthesis code to analyze properties of double compact object binaries as sources of gravitational waves. Since the distribution of lifetimes of these objects extends up to the Hubble time we conclude that a proper calculation of the expected rate must include a full cosmological model. We present such model, calculate the expected coalescence rates, and analyze the intrinsic sensitivity of these rates to the model assumptions. We find that the rate alone is a very poor estimator of the underlying stellar evolution model. However we show that the distribution of observed chirp masses is very sensitive to the underlying stellar evolution model, while it is very insensitive to the underlying cosmology, star formation rate history and variation of detector sensitivity.
\end{abstract}

Key words. gravitational waves - stars: binaries: general

\section{Introduction}

Large interferometric gravitational wave detectors are entering the realm of astronomy: LIGO (Abramovici et al. 1992) has now completed its first two science runs, and is improving its sensitivity, VIRGO (Bradaschia et al. 1990) preparations are proceeding very well. GEO 600 (Danzmann et al. 1992) has achieved an astonishing duty cycle above $97 \%$, and TAMA 300 (Tsubono 1995) has been taking data in coincidence with LIGO. The sensitivity of all the instruments is improving and this leads to increased hopes for detecting gravitational waves. While detection of a gravitational wave will be a huge triumph by itself, the following questions appear: can there be any useful astrophysics done with gravitational waves? Can gravitational wave observations provide any constraints on astrophysical models, and be used to answer questions related to what is known as standard astronomy?

Coalescing compact object binaries are one of the most promising, if not the most promising, sources of gravitational waves. First, we know from electromagnetic observations that they exist and that they emit gravitational waves. Moreover, the known sources will coalesce. The research on the properties of coalescing compact object binaries has concentrated so far on calculation of the expected rates. This problem has been approached in two ways. The first approach was based on analyzing the properties of the existing systems, and considering all the possible selection effects that may affect detectability

Send offprint requests to: T. Bulik, e-mail: bulik@camk. edu.pl of such systems (Narayan et al. 1991; Kalogera et al. 2001). The drawback of this approach is the small number statistics, or even zero object statistics, in the case of black hole neutron star or double black hole binaries. Moreover, the estimates of different selection effects carry additional uncertainty. A second approach is based on studying the stellar evolution processes and detailed analysis of the formation paths of double compact object binaries (Lipunov et al. 1997b; Fryer et al. 1998, 1999; Portegies Zwart \& Yungelson 1998; Bethe \& Brown 1998; Belczyński \& Bulik 1999; Bulik et al. 1999; Belczynski et al. 2002c; Nutzman et al. 2003). Within this approach the main problem is insufficient knowledge of certain processes in stellar evolution. Such processes were parameterized and the results appear to vary significantly with some of this parameters. The uncertainty on the expected rate of compact object coalescences is between two and three orders of magnitude. In our previous paper (Bulik \& Belczyński 2003) we pointed out another possibility: we have shown that measurement of chirp masses of coalescing systems carries a lot of information about the underlying stellar evolution. This calculation used a set of simplifying assumptions: Euclidean space geometry and a constant star formation rate. In this paper we relax these assumptions and consider a more general model. In Sect. 2 we describe the population synthesis model and argue for the need to consider a fully cosmological model of the source distribution to calculate the distributions of observable quantities. Section 3 contains an outline of such calculations, and a discussion of the dependence of the final results on various cosmological parameters and assumptions. We present a discussion of the 
properties of observed chirp mass distribution in Sect. 4, and we finish with conclusions in Sect. 5.

\section{Population synthesis implications}

We use the StarTrack population synthesis code (Belczynski et al. 2002c). The single star evolution in the code is based on the approximate analytical formulae compiled by Hurley et al. (2000), and includes such stages as the main sequence, Hertzsprung gap evolution, red giant branch, core helium burning, asymptotic giant branch, and the helium star evolution. The end product of the stellar evolution can be a white dwarf, a neutron star, or a black hole. The binary evolution description includes such processes as variation of the orbits due to wind mass loss, tides, as well as magnetic breaking, and various mass transfer modes: conservative, quasi dynamic, common envelope evolution. We also take into account the variation of the structure of a star in response to accretion (e.g. rejuvenation) and a possibility of hypercritical accretion onto compact objects. Supernovae explosions are treated in detail using the results of hydrodynamical simulations (Fryer 1999). We take into account both direct and fall back black hole formation. Finally, we investigate various kick velocity distributions. The initial mass of the primary is drawn from a power law distribution $\propto M^{-2.7}$ (Scalo 1986), the initial mass ratio distribution is flat (Kuiper 1935), the eccentricities are drawn from a distribution $\propto e$ (Heggie 1975; Duquennoy \& Mayor 1991), and the orbital separation distribution is flat in $\log a$ (Abt 1983). Apart from the standard model (model A) described in detail by Belczynski et al. (2002c) we also consider a variety of different population synthesis models where we vary parameterizations of stellar evolutionary stages. A list of models used later on is presented in Table 1.

In considering the standard model of population synthesis (model A) we evolved $N_{\text {tot }}=10^{7}$ initial binaries. All other models were run with $N_{\text {tot }}=4 \times 10^{6}$ initial binaries. The number of coalescing compact object binaries obtained in each simulation is listed in Table 1. An example of an evolutionary path leading to formation of a double black hole binary is presented in Fig. 1. In this particular example, the evolution starts with two massive stars (primary mass $M_{1}=53.0 M_{\odot}$, secondary mass $M_{1}=28.4 M_{\odot}$ ) at Zero Age Main Sequence on a rather wide (semi major axis of orbit $a=176 R_{\odot}$ ) and eccentric orbit (eccentricity $e=0.4$ ). The more massive primary evolves first off the Main Sequence (MS) and starts evolution along the giant branch. Due to a rapid radial expansion, it fills its Roche lobe circularizing the orbit and initiates the first mass transfer (MT) episode (stage II). This transfer is stable: it involves little orbital contraction and we assume non-conservative evolution: the primary loses its entire envelope, half of which is lost from the system and only the remaining half is attached to the companion. After the MT episode we are left with the bare helium core of the primary and now a more massive rejuvenated MS secondary (stage III). The primary evolves fast and ends its life in a SN Ib explosion, inducing a slight eccentricity on the binary and widening the orbit (IV). As a result of the core collapse/SN event a first BH is formed (V). Now, the secondary follows the early path of its companion. It leaves the

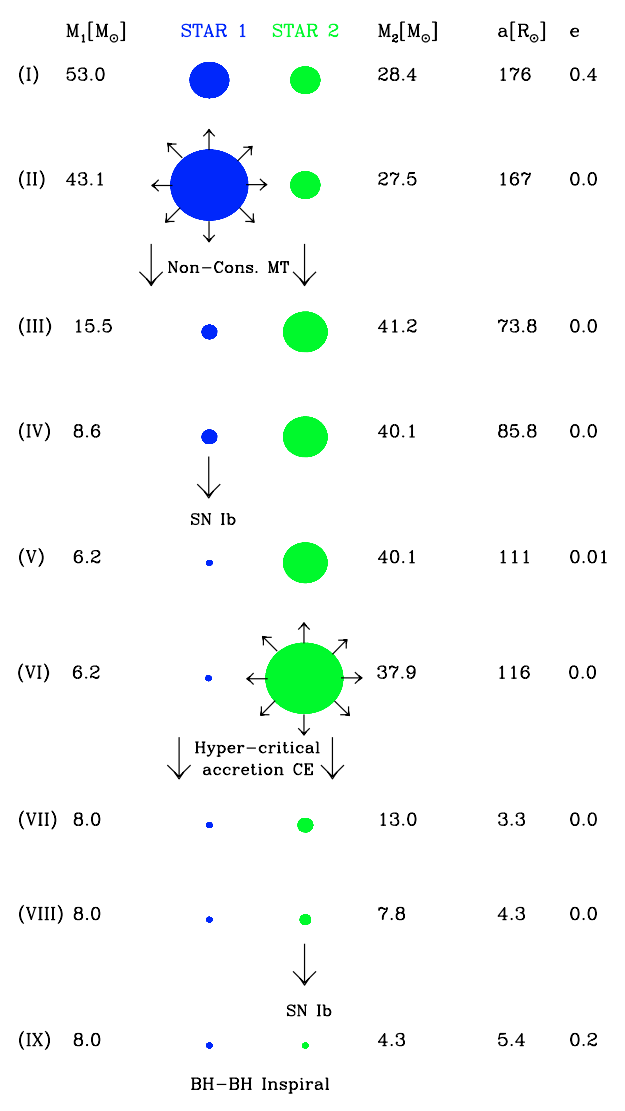

Fig. 1. An example evolutionary scenario leading to formation of a double black hole binary. For details see the text.

MS and becomes a giant. Radial expansion leads to the second MT episode, which due to the extreme mass ratio of the two components (stage VI), is dynamically unstable. The system goes through a Common Envelope (CE) phase, with drastic shrinkage of the orbit, while almost the entire secondary envelope is ejected from the system. A small fraction of the envelope is accreted onto the $\mathrm{BH}$ and the secondary becomes a massive helium star (stage VII). Further nuclear burning of elements in the interior of the helium star brings it finally to a core collapse and SN Ib explosion (stage VIII). The second BH is formed, and we end up with two massive stellar BHs orbiting each other on a tight and eccentric orbit (stage IX).

For each compact object binary we note the masses of individual objects $m_{1}$ and $m_{2}$, and the lifetimes $t_{\text {life }}$ of the system from its creation on ZAMS to formation of the compact object binary and to the merger due to gravitational wave energy loss. Within our code the distinction between a neutron star and a black hole is based solely on its mass. In our calculations we have been assuming a maximum mass of the neutron star $M_{\max }^{\mathrm{NS}}=3 M_{\odot}$; all compact objects heavier than $M_{\max }^{\mathrm{NS}}$ are considered to be black holes. We can distinguish three different types of binaries in our calculations: double neutron star binaries (NSNS), black hole neutron star binaries (BHNS) and double black hole binaries (BHBH).

We plot in Fig. 2 the distribution of the lifetimes of these three types of binaries obtained within model A. The majority of double neutron star binaries are rather short lived, with lifetimes in the range from 10 to 50 Myrs (Belczynski et al. 2002a). 
Table 1. Description of different population synthesis models used here. We list the number of binaries produced in each simulation.

\begin{tabular}{llr}
\hline \hline Model & Description & $N$ produced \\
\hline A & standard model described in Belczynski et al. (2002c), but & 33437 \\
& with $T_{\text {Hubble }}=15$ Gyrs & \\
B1 & zero kicks & 47690 \\
B7 & single Maxwellian with $\sigma=50 \mathrm{~km} \mathrm{~s}^{-1}$ & 47715 \\
B11 & single Maxwellian with $\sigma=500 \mathrm{~km} \mathrm{~s}^{-1}$ & 5246 \\
B13 & Paczynski (1990) kicks with $V_{\mathrm{k}}=600 \mathrm{~km} \mathrm{~s}^{-1}$ & 5247 \\
C & no hyper-critical accretion onto NS/BH in CEs & 8416 \\
E1 & $\alpha_{\mathrm{CE}} \times \lambda=0.1$ & 1003 \\
E2 & $\alpha_{\mathrm{CE}} \times \lambda=0.5$ & 5538 \\
E3 & $\alpha_{\mathrm{CE}} \times \lambda=2$ & 17595 \\
F1 & mass fraction accreted: $f_{\mathrm{a}}=0.1$ & 4461 \\
F2 & mass fraction accreted: $f_{\mathrm{a}}=1$ & 9885 \\
G1 & wind changed by $f_{\text {wind }}=0.5$ & 12345 \\
G2 & wind changed by $f_{\text {wind }}=2$ & 11482 \\
J & primary mass: $\propto M_{1}^{-2.35}$ & 13903 \\
L1 & angular momentum of material lost in MT: $j=0.5$ & 13529 \\
L2 & angular momentum of material lost in $\mathrm{MT}: j=2.0$ & 3335 \\
M1 & initial mass ratio distribution: $\Phi(q) \propto q^{-2.7}$ & 1363 \\
M2 & initial mass ratio distribution: $\Phi(q) \propto q^{3}$ & 22097 \\
O & partial fall back for $5.0<M_{\mathrm{CO}}<14.0 M_{\odot}$ & 9193 \\
S & all systems formed in circular orbits & 8306 \\
Z1 & metallicity: $Z=0.01$ & 8520 \\
Z2 & metallicity: $Z=0.0001$ & 10481 \\
\hline & &
\end{tabular}

Low mass helium stars $\left(\leq 4 M_{\odot}\right)$, which may eventually produce NSs but not BHs, undergo a significant expansion and may possibly initiate a MT episode in the formation of compact object binaries (Belczyński \& Kalogera 2001; Belczynski et al. 2002a; Dewi et al. 2002; Ivanova et al. 2003). The RLOF (either dynamically stable or unstable) initiated in the progenitor system with a low mass helium star donor and a compact companion may effectively reduce the binary separation. Since only double neutron star progenitors are affected by the above process the final separations and thus merger times of compact object binaries consisting of two neutron stars are much shorter than those of black hole binaries. The mixed BHNS binary lifetimes span a very wide range from roughly $10 \mathrm{Myrs}$ until the Hubble time with no preferred interval. The BHBH binaries live much longer. Their lifetimes extend from about $100 \mathrm{Myrs}$ to the Hubble time. Thus the currently merging NSNS binaries originate in stars formed in relatively recent starbursts, while the BHBH and BHNS binaries originate in star that have formed a few billion years ago. Lipunov et al. (1997a) and recently Bulik \& Belczyński (2003) have shown that the observed sample of coalescing binaries is dominated by the BHBH binaries. Since they are so long lived and the star formation rate was probably much higher in the early Universe the accurate calculation of their observed properties requires taking into account a full cosmological of distribution and evolution of such sources.

\section{Expected numbers of observable mergers}

The signal to noise ratio from a coalescence of a binary in gravitational wave interferometers has been calculated by several authors (Chernoff \& Finn 1993; Bonazzola \& Marck 1994;

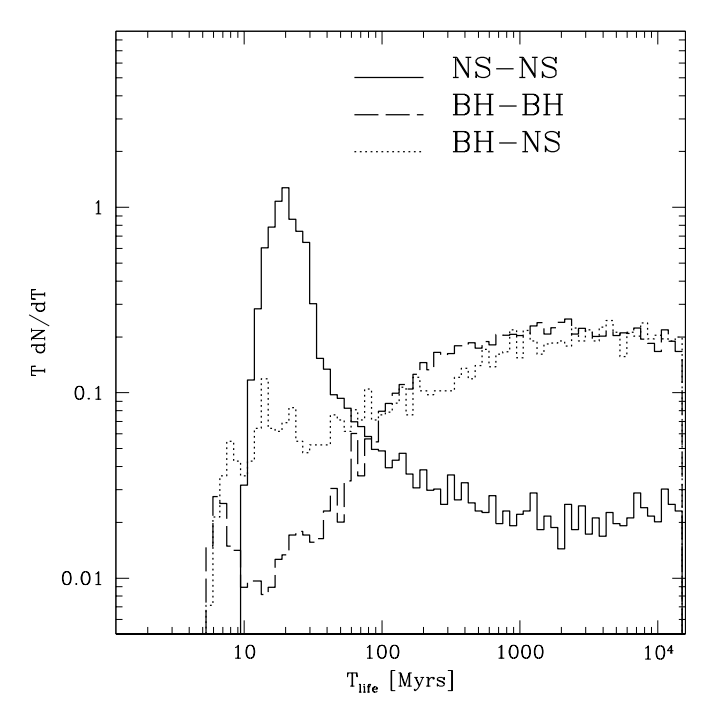

Fig. 2. Distribution of the lifetimes of the double neutron star binaries (solid line), black hole neutron star binaries (dotted line), and double black hole binaries (dashed lines).

Flanagan \& Hughes 1998). In the frequency region where the detectors are most sensitive it is

$(S / N)=\frac{A_{i}}{d_{\mathrm{L}}}\left[\frac{(1+z) \mathcal{M}}{M_{\odot}}\right]^{5 / 6}$,

where $\mathcal{M}=\left(m_{1} m_{2}\right)^{0.6}\left(m_{1}+m_{2}\right)^{-0.2}$ is the chirp mass, $z$ is the redshift, $d_{\mathrm{L}}$ is the luminosity distance, and the $A_{i}$ depends on the details of a particular detector. In a more detailed calculation - see Flanagan \& Hughes (1998) for the cases of LIGO I, LIGO II, and LISA - Eq. (1) becomes more complex due to the 


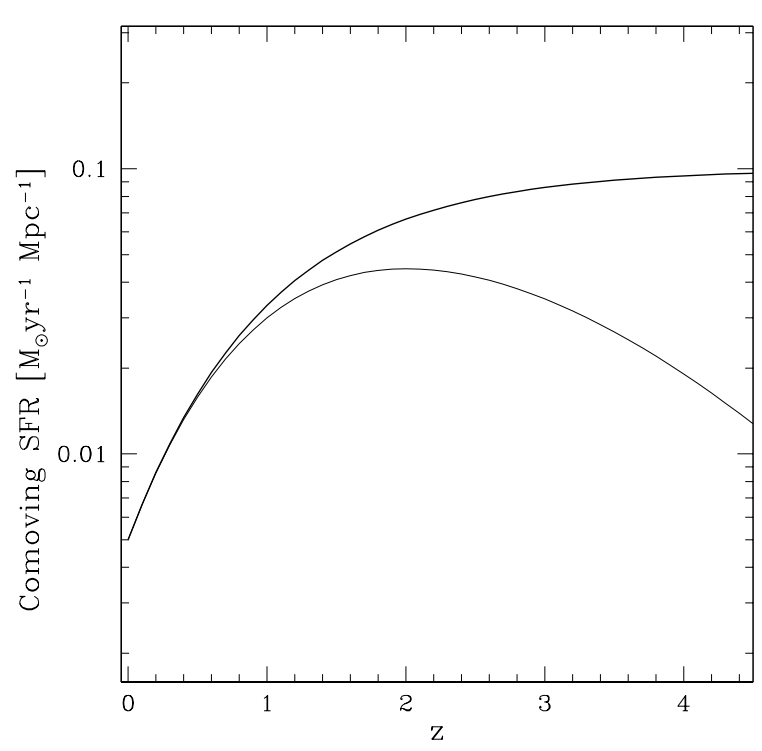

Fig. 3. The star formation rate history models used here: thin line (Madau et al. 1996), thick line (Rowan-Robinson 1999).

finite size of the frequency interval available for a given detector. This formula is valid with an accuracy better than 10 percent for chirp masses below $\mathcal{M}<20 M_{\odot}$. In the following calculations we assume a flat cosmological model with the matter density $\Omega_{\mathrm{m}}=0.3$, and cosmological constant $\Omega_{\Lambda}=0.7$, the Hubble constant $H_{0}$ is $65 \mathrm{~km}^{-1} \mathrm{Mpc}^{-1}$. We assume the model of star formation history rate presented as a thick solid line in Fig. 3 (Rowan-Robinson 1999), but we also consider a model in which the star formation rate ceases at $z>2-$ thin line in Fig. 3 (Madau et al. 1996), and a flat star formation rate.

Finding the sampling redshift $z_{\mathcal{M}}$ requires solving the equation

$d_{\text {prop }}\left(z_{\mathcal{M}}\right)\left(1+z_{\mathcal{M}}\right)^{1 / 6}=A_{i}\left(\mathcal{M} / M_{\odot}\right)^{5 / 6}$

where $d_{\mathrm{L}}=(1+z) d_{\text {prop }}$ and

$d_{\text {prop }}(z)=\int_{0}^{z}(1+z) c\left|\frac{\mathrm{d} t}{\mathrm{~d} z}\right| \mathrm{d} z$

is the proper distance, and we have introduced the cosmic time $t$ :

$\left|\frac{\mathrm{d} t}{\mathrm{~d} z}\right|=\frac{1}{H_{0}(1+z)\left[\left(\Omega_{\mathrm{m}}(1+z)^{3}+\left(1-\Omega_{\mathrm{m}}\right)\right]^{1 / 2}\right.}$,

in the flat space time.

Formation of compact object binaries is directly related to the star formation rate. After a binary is formed it evolves for a time $t_{\text {life }}$ until its merger. This time includes the stellar evolutionary time needed to form a compact object binary and then the life time of such binary due to gravitational wave emission. The coalescence is delayed by $t_{\text {life }}$ with respect to the star formation. Let us denote by $F(\mathcal{M}, t, z)$ the formation rate of binaries with the chirp mass $\mathcal{M}$ and a lifetime $t$ as a function of redshift. The star formation rate history in the Universe $S F R(z)$ can now be combined with the results of the population synthesis code to find $F(\mathcal{M}, t, z)$. To this end we need two quantities: the average stellar mass $\left\langle M_{*}\right\rangle$, so that we find the star number formation rate $S F R(z) /\left\langle M_{*}\right\rangle$, and the fraction of the stellar population that we simulate with the stellar population synthesis code $f_{\text {sim }}$, to obtain the number formation rate of our binaries as a function of $z: S F R(z) f_{\text {sim }} /\left\langle M_{*}\right\rangle$. For the assumed slope of the initial mass function $\alpha=-2.7$, the minimal stellar mass of $0.2 M_{\odot}$, maximal stellar mass of $100 M_{\odot}$, and binary fraction of 0.5 , the average stellar mass is $\left\langle M_{*}\right\rangle=0.87 M_{\odot}$, and the fraction of stars that we simulate is $f_{\text {sim }}=1.24 \times 10^{-3}$. The numerical estimate of the formation rate of binaries in a given interval between $\mathcal{M}$ and $\mathcal{M}+\mathrm{d} \mathcal{M}$, with lifetimes between $t$ and $t+\mathrm{d} t$ is

$F(\mathcal{M}, t, z) \mathrm{d} \mathcal{M} \mathrm{d} t=S F R(z) \frac{f_{\text {sim }}}{\left\langle M_{*}\right\rangle} \frac{N_{i}}{N_{\text {tot }}}$

where $N_{i}$ is the number of binaries in a simulation with the chirp mass and the lifetime in a given interval and $N_{\text {tot }}$ is the total number of simulated binaries.

Binaries coalescing at a given redshift $z_{0}$ originate from binaries formed at different earlier times. The rate of coalescences of binaries with a given chirp mass $\mathcal{M}$ is then given by

$\frac{\mathrm{d} f_{\text {coal }}\left(z_{0}\right)}{\mathrm{d} \mathcal{M}}=\int \mathrm{d} t^{\prime} F\left(\mathcal{M}, t^{\prime}, z_{\mathrm{f}}\right)$,

where the source formation redshift $z_{\mathrm{f}}$, is obtained by solving:

$t^{\prime}=\int_{z_{0}}^{z_{\mathrm{f}}}\left|\frac{\mathrm{d} t}{\mathrm{~d} z}\right| \mathrm{d} z$

We can now proceed to calculation of the observed rate of coalescences. This calculation is very similar to the ones performed in the case of gamma-ray bursts (Totani 1999; Belczynski et al. 2002b). An instrument can detect a signal characterized by the chirp mass $\mathcal{M}$ if it lies closer than the sampling redshift $z_{\mathcal{M}}$. The rate at which such an instrument will detect binary coalescences is

$\frac{\mathrm{d} R}{\mathrm{~d} \mathcal{M}}=\int_{0}^{z_{\mathcal{M}}} \frac{\mathrm{d} f_{\text {coal }}(z)}{\mathrm{d} \mathcal{M}} \frac{1}{1+z} \frac{\mathrm{d} V}{\mathrm{~d} z} \mathrm{~d} z$

where

$\frac{\mathrm{d} V}{\mathrm{~d} z}=4 \pi c \frac{d_{\mathrm{L}}^{2}}{(1+z)}\left|\frac{\mathrm{d} t}{\mathrm{~d} z}\right|$

is the comoving volume element.

The results of the differential rate calculations are shown in Fig. 4 where we present the model $\frac{\mathrm{d} R}{\mathrm{~d} \mathcal{M}}$ requiring a signal to noise $(S / N)>8$ and using the sensitivities calculated by Flanagan \& Hughes (1998), LIGO I and LIGO II and by Hello (1998) for VIRGO, i.e.: $A_{\mathrm{LIGO} 1}=100 \mathrm{Mpc}, A_{\mathrm{VIRGO}}=$ $150 \mathrm{Mpc}$, and $A_{\mathrm{LIGO} 1}=2200 \mathrm{Mpc}$. There are two main effects influencing the $\frac{\mathrm{d} R}{\mathrm{~d} \mathcal{M}}$ presented in Fig. 4: the first is that with increasing sensitivity a detector detects coalescences from a larger volume and the second is due to the fact that the observed quantity is the redshifted chirp mass $(1+z) \mathcal{M}$. A highly sensitive detector like LIGO 2 should detect coalescing binaries at non negligible redshifts and this leads to the spread of the distribution of observed chirp masses to higher values.

We note the uncertainties in the estimates shown in Fig. 4, especially coming from the product $S F R(z) f_{\text {sim }}\left\langle M_{*}\right\rangle^{-1}$. The estimates of the star formation rates are uncertain by a factor of 


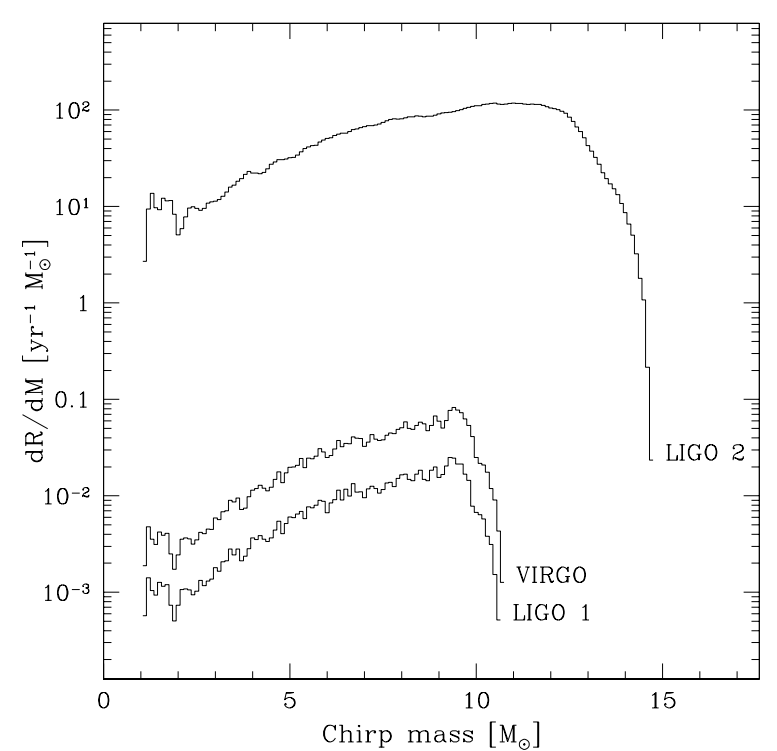

Fig. 4. The differential detection rates expected for the existing long base interferometers.

at least two (Madau et al. 1996). Both $f_{\text {sim }}$ and $\left\langle M_{*}\right\rangle$ depend strongly on the assumed slope of the initial mass function and the lower mass cutoff of the stellar population and weakly on the binary fraction. The low mass stars, not taken into account in our simulations since they do not lead to compact object binaries, dominate the stellar population in number and in mass. We have assumed a power law shape of the initial mass function, yet the absolute numbers of the high mass progenitors of compact object binaries will be affected by deviation of the initial mass function from a simple power law. A deviation of the slope by $1 / 2$ changes the number of high mass stars in relation to the total population by a factor of ten. In summary in the calculation of the rate using Eq. (8), there are at least the following uncertainties: the unknown binary fraction, that could be as high as a factor of two, the unknown shape of the initial mass function leads to uncertainty by a factor of up to ten. The differences between the galactic coalescence rates obtained in the framework of various models of stellar evolution (Belczynski et al. 2002c) amount to a factor of about \pm 30 . The difference in the expected rate within various stellar evolution models is comparable to the intrinsic uncertainty in the rate. Thus, given a measurement of rate, answering an inverse problem, i.e. what does the rate tell us about the underlying stellar evolution is not possible. Here I have assumed that the measured rate has no uncertainty, yet we know that the interferometric detectors exhibit a very non stationary noise which hampers an accurate estimate of the timespace volume surveyed.

\section{Distribution of chirp masses}

We note that the shape of the observed distributions of chirp masses in Fig. 4 is nearly identical for the LIGO 1 and VIRGO detectors. It is therefore interesting to consider the distribution of the observed chirp masses as a potential observational statistic. One obvious advantage of such a distribution is that all the problems concerning the rate vanish. The uncertainties in the normalization that entered the calculation of the rate vanish
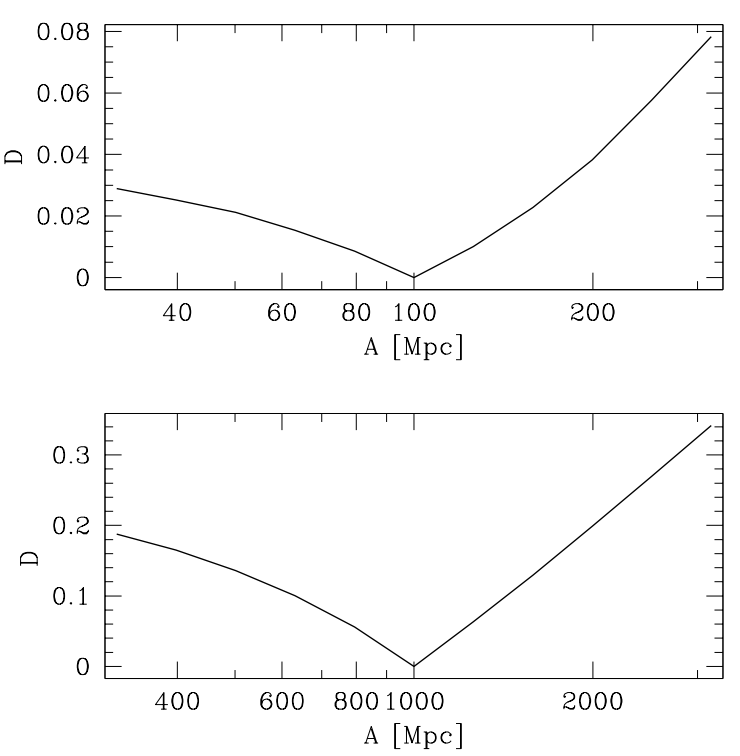

Fig. 5. Variation of the shape of the distribution of observed chirp masses with the sensitivity of a detector. The top panel corresponds to the reference model one, and the bottom panel to the reference model two. On the vertical axis we plot the Kolmogorov Smirnov parameter $D$ comparing a given distribution with the standard model described in the text.

when the distributions of observed chirp masses are considered. We now consider the sensitivity of the distribution of observed chirp masses to the assumed cosmological model, and to the sensitivity of the detector. Finally we will present the sensitivity of this statistic to the assumed model of stellar evolution.

We define two reference models with which other models will be compared. These models corresponds to the cosmological model described in Sect. 2, with the star formation rate given by the thick line in Fig. 3, and the sensitivity of the detector given by Eq. (1), with $A_{1}=100 \mathrm{Mpc}$ for model one and $A_{2}=1 \mathrm{Gpc}$ for model two. In the following we will use a simple method of comparing distributions: the Kolmogorov Smirnov test. This test uses a parameter $D$ defined as the maximum distance between two cumulative distributions. Two distributions differing by $D$ can be distinguished at a confidence level of approximately $10^{-4}$, when they are sampled at $N \approx 4 / D^{2}$ points.

We first analyze the dependence of the expected distribution of observed chirp masses on detector sensitivity. Variation in the sensitivity of a detector is modeled by the parameter $A_{i}$ in Eq. (1). We present the results in Fig. 5 for our two reference models. For the case of the reference model one (top panel of Fig. 5), the maximum value of the parameter $D$ reaches 0.04 when the sensitivity is increased or decreased by $50 \%$. For the more sensitive detector - reference model two in the bottom panel of Fig. 5 - the difference is larger and the parameter $D$ reaches the value of 0.15 . This is due to the fact that the redshift effects play a much stronger role in the more sensitive detectors. A smaller effects occurs because the detector starts seeing NSNS mergers from redshifts where the star formation rate was larger than the local one.

We present the dependence of the shape of the distribution of observed chirp masses on the assumed cosmological 


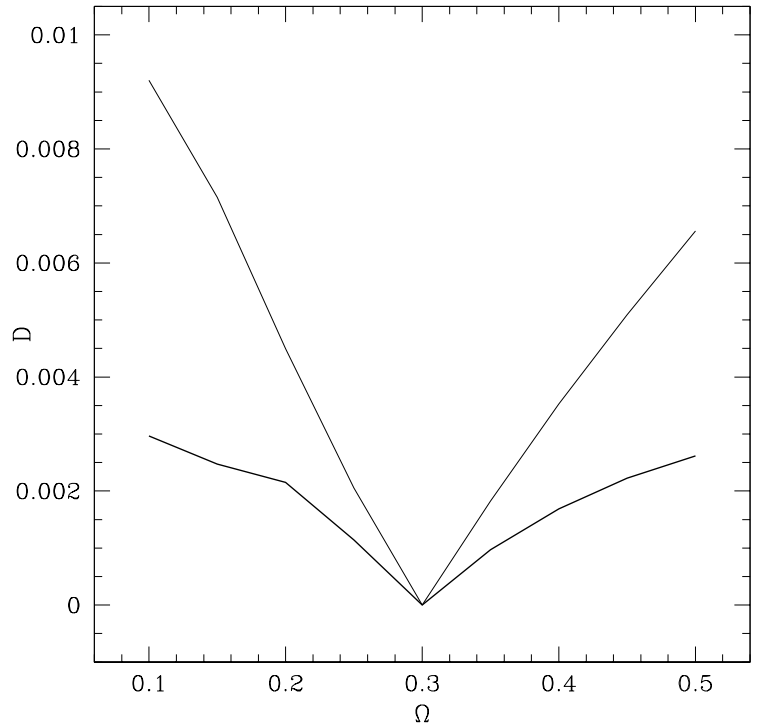

Fig. 6. Change in the distribution of the observed chirp masses as a function of the assumed mass density in the cosmological model. The thick line corresponds to the reference model one, and the thin line to the reference model two.

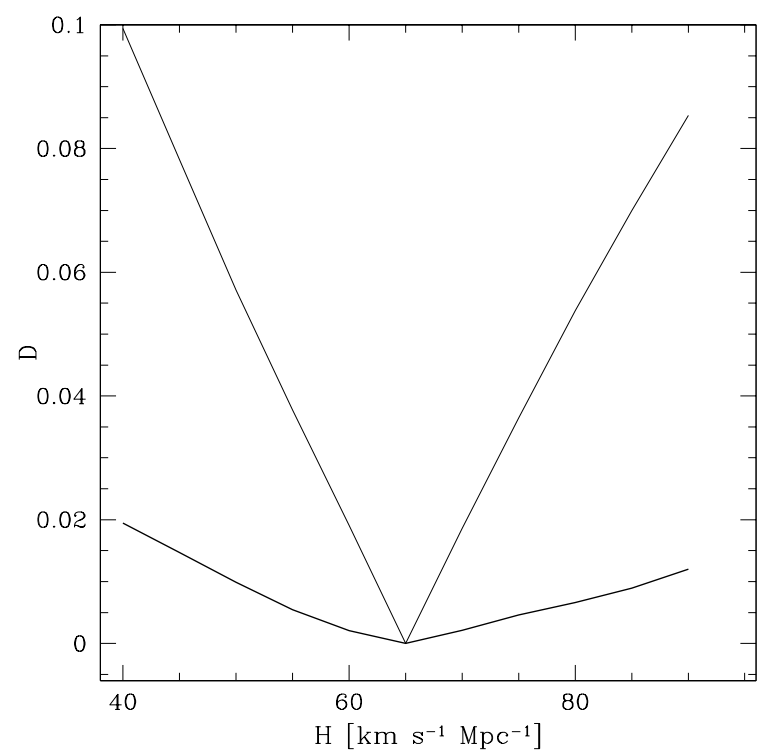

Fig. 7. Change in the distribution of the observed chirp masses as a function of the assumed value of the Hubble constant. The thick line corresponds to the reference model one, and the thin line to the reference model two.

model in Figs. 6 and 7. The thick lines denote reference model one with $A_{1}=100 \mathrm{Mpc}$, and we present reference model two $A_{2}=1 \mathrm{Gpc}$ with thin lines. Varying $\Omega_{\mathrm{m}}$ (while keeping the Universe flat, i.e. $\Omega_{\Lambda}=1-\Omega_{\mathrm{m}}$ ) does not alter the shape of the observed distribution significantly: the parameter $D$ never exceeds 0.01 for model two and less than 0.003 for model one. The assumed value of the Hubble constant has a stronger effect, however. When $H_{0}$ is varied by $20 \%$ the parameter $D$ does not exceed 0.01 for model one and 0.04 for model two. We have also investigated the dependence of the distribution of observed chirp masses on the assumed shape of the star formation rate history. Apart from the standard model, with the star formation

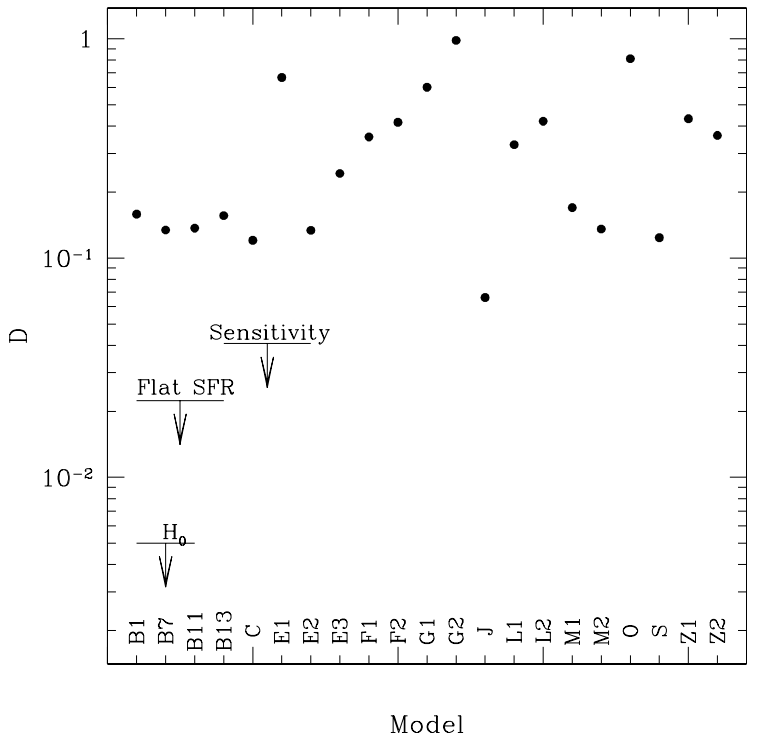

Fig. 8. The dependence of the parameter $D$ comparing the distribution of observed chirp masses of the reference model one with other models of stellar evolution. For comparison we also present the maximal values of $D$ obtained when cosmological parameters are changed, for different star formation models, and when the sensitivity of the detector varies.

rate history given by the thick line in Fig. 3, we investigated a model where the star formation rate ceases above $z=2$, shown as a thick line in Fig. 3, and a model where the star formation rate is constant. The differences between the resulting distribution for model one and the model with star formation decreasing above $z=2$ leads to $D=0.011$, and for the flat star formation rate we obtained $D=0.021$, while for model two these differences are: $D=0.010$ and $D=0.036$. Such a weak dependence on the star formation rate is not difficult to understand. The distribution of observed chirp masses is dominated by the binaries with the largest chirp masses - the BHBH binaries, while the NSNS binaries constitute only a small fraction of the observed systems. Since the NSNS binaries are shortlived, they originate in stars born recently and their number does not depend on the star formation history at high redshifts. The star formation history affects only the number of the high chirp mass $(\mathrm{BHBH})$ binaries, however they already dominate the observed sample. Therefore models with different star formation rate histories vary by the number of NSNS binaries with respect to the $\mathrm{BHBH}$ binaries, but all of the models are dominated by the heavy BHBH binaries. The resulting differences in the normalized distribution of observed chirp masses is therefore small.

We summarize the results of this section in Figs. 8 and 9. Here we plot the values of the parameter $D$ obtained from comparing our models one (Fig. 8), and two (Fig. 9) with distributions obtained when the stellar evolution is described by models listed in Table 1. We also present the maximal values of the parameter $D$ obtained when we varied the detector sensitivity, the cosmological model, and also for different assumed forms of star formation rate history. For most of the models, the value of $D$ lies between 0.1 and unity. The exception is model $\mathrm{J}$ for which $D=0.07$. This is the model with a different slope of 


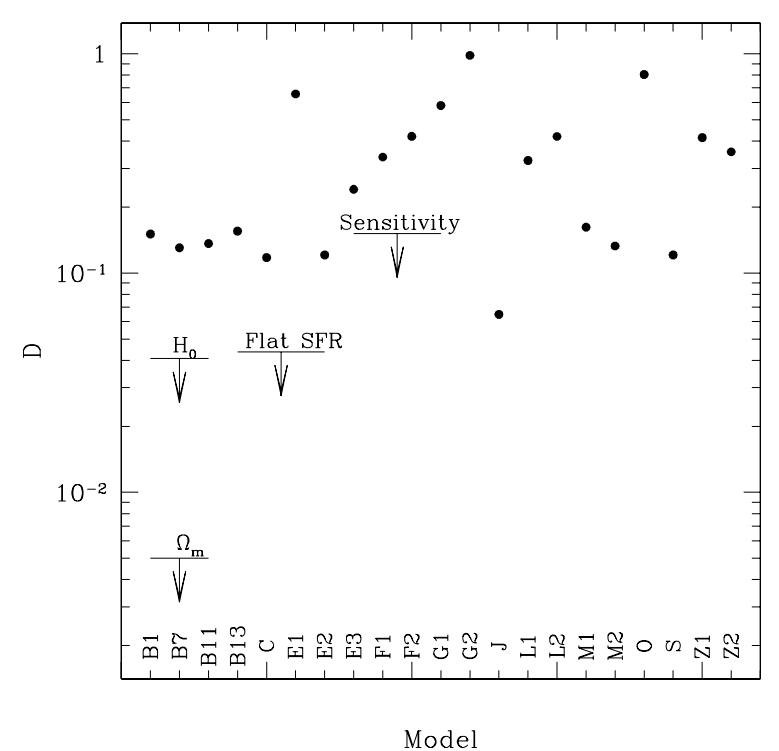

Fig. 9. Same as Fig. 8 but for reference model two.

the initial mass function. The parameter $D$ is small in this case since the distribution of observed chirp masses is dominated by heavy double black hole mergers. Decreasing the slope of the initial mass function only enhances this effect, since it increases the ratio of black hole formation rate to neutron star formation rate. For a number of models E1, G1, G2, and O, the parameter $D$ is larger than 0.5 . These models vary from the standard one by parameters that affect the masses of compact object the most. The remaining models have the $D$ parameter in the range between 0.1 and 0.5 . Distributions differing by $D=0.2$ can be distinguished when sampled with approximately one hundred points. Thus we confirm our previous conclusion (Bulik \& Belczyński 2003) that observation of about 100 mergers can yield significant constraints on the models of evolution of high mass stars. In Fig. 8 the expected distribution of chirp masses is mostly influenced by the underlying stellar evolution model, while in the case of model two shown in Fig. 9, variation of the sensitivity of the detector may influence the shape of the chirp mass distribution at a level similar to several stellar models. Thus we conclude that the distribution of observed chirp masses is a rather robust estimator of the underlying stellar evolution.

\section{Summary}

Using the StarTrack binary population synthesis code we have investigated the properties of the population of double compact objects - the primary candidate sources of gravitational waves for high frequency interferometric detectors. We find that the distribution of lifetimes of various types of binaries is different: double neutron star binaries live typically for a few tens of million years, while the lifetimes of binaries containing black holes extend up to the Hubble time. Therefore, the double black hole binaries merging currently in our Milky way neighborhood originate in systems formed in early star formation episodes. Hence, a proper calculation of the number and properties of the merging compact object binaries should include the full cosmological model of formation of these sources. We outline such calculation and calculate the expected differential rate per unit observed chirp mass. We discuss the usefulness of the observed rate for determining the properties of the underlying population. We estimate the systematic uncertainty of the calculation of the rates due to uncertainty in the model. We find that the rate calculation carries a huge systematic error, comparable to the spread in the rate due to variation of the stellar evolution model. We conclude that it is very unlikely that significant constraints can be obtained from consideration and modeling of the rate alone. However, we analyze the properties of another observable, the distribution of observed chirp masses. We show that is very insensitive to the parameters that made the rate estimate uncertain. Moreover, we test the sensitivity of this statistic to the assumed parameters of the cosmological model: the mass density in the Universe, the value of the Hubble constant, and the model of star formation rate. All of these parameters hardly affect the shape of the distribution of observed chirp masses. We also verify that the shape of this distribution does not vary significantly when the detector sensitivity changes. This is especially important for detectors with non-stationary noise. Non-stationary noise would make comparison of the theoretical rate with observations even more uncertain and difficult, yet it poses no problem for the analysis of the distribution of observed chirp masses. We find that the most important factor determining the shape of the observed distribution of chirp masses is the underlying stellar evolution model. Different stellar models lead to different masses of compact objects in compact object binaries. The strength of the winds affects the masses of the stellar cores at the time of supernovae explosions; differences in mass transfer prescriptions lead to differences in the amount of mass accreted and hence leads to different masses; different assumed fall back masses are present at SN explosion, which strongly affects masses of newly born compact objects (see also discussion in Bulik \& Belczyński 2003). Analysis of the distributions of the observed chirp masses is therefore a very valuable tool to use the gravitational wave data to impose constraints on the stellar evolution models.

The changes of the distribution of observed chirp masses with varying detector sensitivity are mainly due to the fact that the observed quantity is the redshifted chirp mass. Yet a redshift of a coalescing source may be measured provided that we know its location (Finn \& Chernoff 1993). The location can be estimated using a network of gravitational wave detectors. A measurement of the redshift would lead to an estimate of the chirp mass, and to removal of the bulk of the dependence of the observed chirp mass distribution on the fluctuations of detector sensitivity.

We note that in general each measurement of a coalescence may also carry more information, like e.g. the individual masses of the coalescing object or their spins. Once these become available they must be included in the analysis. In this paper we have considered only the chirp mass measurement, as this is the most conservative approach.

Acknowledgements. This research was supported by the $\mathrm{KBN}$ grant 5P03D01120. 


\section{References}

Abramovici, A., Althouse, W. E., Drever, R. W. P., et al. 1992, Science, 256, 325

Abt, H. A. 1983, ARA\&A, 21, 343

Belczyński, K., \& Bulik, T. 1999, A\&A, 346, 91

Belczyński, K., \& Kalogera, V. 2001, ApJ, 550, L183

Belczynski, K., Bulik, T., \& Kalogera, V. 2002a, ApJ, 571, L147

Belczynski, K., Bulik, T., \& Rudak, B. 2002b, ApJ, 571, 394

Belczynski, K., Kalogera, V., \& Bulik, T. 2002c, ApJ, 572, 407

Bethe, H. A., \& Brown, G. E. 1998, ApJ, 506, 780

Bonazzola, S., \& Marck, J. A. 1994, Annu. Rev. Nucl. Part. Sci., 45, 655

Bradaschia, et al. 1990, Nucl. Instr. Meth., A289, 518

Bulik, T., \& Belczyński, K. 2003, ApJ, 589, L37

Bulik, T., Belczyński, K., \& Zbijewski, W. 1999, MNRAS, 309, 629

Chernoff, D. F., \& Finn, L. S. 1993, ApJ, 411, L5

Danzmann, K., \& et al. 1992, in Relativistic Gravity Research, Proc. 81 WE-Heraeus-Seminar Held at the Physikzentrum Bad Honnef, Germany, 2-6 September 1991, ed. J. Ehlers, \& G. Schäfer, Also Lecture Notes in Physics (Berlin, Heidelberg, New York: Springer-Verlag), 410, 184

Dewi, J. D. M., Pols, O. R., Savonije, G. J., \& van den Heuvel, E. P. J. 2002, MNRAS, 331, 1027

Duquennoy, A., \& Mayor, M. 1991, A\&A, 248, 485

Finn, L. S., \& Chernoff, D. F. 1993, Phys. Rev. D, 47, 2198

Flanagan, É. É., \& Hughes, S. A. 1998, Phys. Rev. D, 57, 4535
Fryer, C., Burrows, A., \& Benz, W. 1998, ApJ, 496, 333

Fryer, C. L. 1999, ApJ, 522, 413

Fryer, C. L., Woosley, S. E., \& Hartmann, D. H. 1999, ApJ, 526, 152

Heggie, D. C. 1975, MNRAS, 173, 729

Hello, P. 1998, in Proc. Second workshop on Gravitational wave Data Analysis (Paris: Éditions Frontières), 87

Hurley, J. R., Pols, O. R., \& Tout, C. A. 2000, MNRAS, 315, 543

Ivanova, N., Belczynski, K., Kalogera, V., Rasio, F. A., \& Taam, R. E. 2003, ApJ, 592, 475

Kalogera, V., Narayan, R., Spergel, D. N., \& Taylor, J. H. 2001, ApJ, 556, 340

Kuiper, G. P. 1935, PASP, 47, 15

Lipunov, V. M., Postnov, K. A., \& Prokhorov, M. E. 1997a, New Astron., 2, 43

Lipunov, V. M., Postnov, K. A., \& Prokhorov, M. E. 1997b, MNRAS, 288, 245

Madau, P., Ferguson, H. C., Dickinson, M. E., et al. 1996, MNRAS, 283,1388

Narayan, R., Piran, T., \& Shemi, A. 1991, ApJ, 379, L17

Nutzman, P., Kalogera, V., \& Belczynski, K. 2003, in preparation

Paczynski, B. 1990, ApJ, 348, 485

Portegies Zwart, S. F., \& Yungelson, L. R. 1998, A\&A, 332, 173

Rowan-Robinson, M. 1999, Ap\&SS, 266, 291

Scalo, J. M. 1986, Fund. Cosmic Phys., 11, 1

Totani, T. 1999, ApJ, 511, 41

Tsubono, K. 1995, in First Edoardo Amaldi Conference on Gravitational Wave Experiments, 112 